Wright State University

CORE Scholar

$12-1-2002$

\title{
Recombination of Excitons Bound to Oxygen and Silicon Donors in Freestanding GaN
}

\author{
A. Wysmolek \\ K. P. Korona \\ R. Stepniewski \\ J. M. Baranowsky \\ J. Bloniarz
}

See next page for additional authors

Follow this and additional works at: https://corescholar.libraries.wright.edu/physics

Part of the Physics Commons

\section{Repository Citation}

Wysmolek, A., Korona, K. P., Stepniewski, R., Baranowsky, J. M., Bloniarz, J., Potemski, M., Jones, R. L., Look, D. C., Kuhl, J., Park, S. S., \& Lee, S. K. (2002). Recombination of Excitons Bound to Oxygen and Silicon Donors in Freestanding GaN. Physical Review B, 66 (24), 245317.

https://corescholar.libraries.wright.edu/physics/205

This Article is brought to you for free and open access by the Physics at CORE Scholar. It has been accepted for inclusion in Physics Faculty Publications by an authorized administrator of CORE Scholar. For more information, please contact library-corescholar@wright.edu. 


\section{Authors}

A. Wysmolek, K. P. Korona, R. Stepniewski, J. M. Baranowsky, J. Bloniarz, M. Potemski, R. L. Jones, David C. Look, J. Kuhl, S. S. Park, and S. K. Lee 


\title{
Recombination of excitons bound to oxygen and silicon donors in freestanding GaN
}

\author{
A. Wysmolek,,${ }^{1,2, *}$ K. P. Korona,${ }^{1}$ R. Stępniewski, ${ }^{1}$ J. M. Baranowski, ${ }^{1}$ J. Błoniarz, ${ }^{1}$ M. Potemski, ${ }^{2}$ R. L. Jones, ${ }^{3}$ \\ D. C. Look, ${ }^{4}$ J. Kuhl, ${ }^{5}$ S. S. Park, ${ }^{6}$ and S. K. Lee ${ }^{6}$ \\ ${ }^{1}$ Institute of Experimental Physics, Warsaw University, Hoża 69, 00-681 Warsaw, Poland \\ ${ }^{2}$ Grenoble High Magnetic Field Laboratory, MPI/FKF-CNRS, BP166X, F-38042 Grenoble Cedex 9, France \\ ${ }^{3}$ Air Force Research Laboratory, AFRL/MLPS, Wright-Patterson AFB, Ohio \\ ${ }^{4}$ Semiconductor Research Center, Wright State University, Dayton, Ohio \\ ${ }^{5}$ Max-Planck-Institut für Festkörperforschung, Heisenbergstrasse 1, D-70569 Stuttgart, Germany \\ ${ }^{6}$ Samsung Advanced Institute of Technology, P.O. Box 111, Suwon, Korea
}

(Received 27 July 2002; published 26 December 2002)

\begin{abstract}
The neutral donor bound exciton recombination processes in freestanding GaN have been studied. The photoluminescence spectrum shows emission lines related to silicon and oxygen donors. Time-resolved luminescence allows us to correlate the principal donor bound exciton lines with their two-electron satellites. The magnetic field splitting of the two-electron lines is well described by the theory of the hydrogen atom in a magnetic field. For the oxygen donor a $1.5 \mathrm{meV}$ chemical shift and a $30.8 \mathrm{meV}$ effective Rydberg have been evaluated. Two-electron satellites involving excitations to the $2 p$ and $2 s$ donor states are separated by an energy of 1.0 and $1.3 \mathrm{meV}$ for $\mathrm{O}$ and $\mathrm{Si}$ impurity, respectively. The temperature dependence of the two-electron emission clearly shows that this separation arises from a splitting of the ground state of the neutral donor bound exciton complex. The nature of this splitting is discussed and it is suggested that it is due to rotational states of donor bound excitons.
\end{abstract}

DOI: 10.1103/PhysRevB.66.245317

PACS number(s): 78.55.Cr, 71.35.Ji, 71.55.-i

\section{INTRODUCTION}

Freestanding $\mathrm{GaN}$ wafers, i.e., wafers grown on a sapphire substrate and then separated from the wafer, have recently demonstrated the highest mobility ever measured in bulk $\mathrm{GaN}^{1}$ as well as excellent optical and structural properties. $^{2,3}$ However, many of the sharp photoluminescence (PL) lines that appear have not been identified, as yet. In this work, we use time-resolved PL and high-field magnetospectroscopy to study the donor-bound excitons and their two-electron satellites.

A two-electron satellite (TES) transition occurs when an exciton bound to a neutral donor $\left(D^{0} X\right)$ recombines and leaves the donor in an excited state. The two-electron energy is therefore lower than that of the $D^{0} X$ peak by the difference in the ground and excited state energies of the neutral donor. The recombination of an exciton combined with the excitation of a donor has been observed in GaP (Ref. 4) and then for $\mathrm{CdS},{ }^{5} \mathrm{CdSe},{ }^{6} \mathrm{ZnO},{ }^{7}$ and $\mathrm{InP}^{8}{ }^{8}$ The two-electron transition in $\mathrm{GaN}$ has also been reported previously. ${ }^{9-11}$ This transition has been detected in heteroepitaxial layers grown on $\mathrm{Al}_{2} \mathrm{O}_{3}$ (Ref. 9) and in homoepitaxial layers grown on GaN single crystals. ${ }^{10-12}$ The identification of the twoelectron line was confirmed by magneto-optical measurements. ${ }^{13,14}$ However, the nature of the donor involved in the two-electron transitions has not been identified.

In this work we have undertaken a study of the twoelectron transitions in freestanding GaN material using timeresolved and magneto-optic spectroscopy in magnetic fields reaching $28 \mathrm{~T}$. The relatively narrow emission lines in freestanding GaN combined with high magnetic fields allows a more detailed understanding of the PL results.

\section{EXPERIMENTAL DETAILS}

The freestanding GaN sample was grown, separated, and polished at the Samsung Advanced Institute of Technology. The original thickness was about $500 \mu \mathrm{m}$, and the final thickness, after polishing, about $250 \mu \mathrm{m}$. All measurements were performed on the $\mathrm{Ga}$ face, which is the face farthest from the original $\mathrm{Al}_{2} \mathrm{O}_{3}$ substrate, and thus the face with the highest quality material. Room temperature carrier concentration and mobility measured on a similar sample were 5 $\times 10^{15} \mathrm{~cm}^{-3}$ and $1240 \mathrm{~cm}^{2} / \mathrm{V} \mathrm{s}$, respectively.

The magneto-optical measurements were carried out by using a resistive magnet, in the Grenoble facility. Luminescence was excited with a He-Cd laser and spectra were acquired at liquid-helium temperature with a CCD camera. The spectral resolution in the magneto-optic experiment was about $0.2 \mathrm{meV}$.

The time-resolved PL measurements were performed with frequency-doubled output pulses (150 fs) from a modelocked Ti:sapphire laser. The spectral and temporal distributions of the PL were analyzed with a streak camera (20 ps resolution) and a monochromator ( $0.6 \mathrm{meV}$ resolution).

\section{RESULTS}

The emission spectrum of freestanding GaN at low temperature in the donor bound exciton region is shown in Fig. 1. Clearly three emission lines $D_{1}^{0} X_{A}, D_{2}^{0} X_{A}$ and $D_{3}^{0} X_{A}$ connected with the upper valence band (A) are found at 3.4710, 3.4720 , and $3.4729 \mathrm{eV}$, respectively. That indicates that there are three donors in this material capable of binding excitons. The emission line corresponding to the free exciton A recombination is observed at $3.4789 \mathrm{eV}$ (not shown in Fig. 1), what 


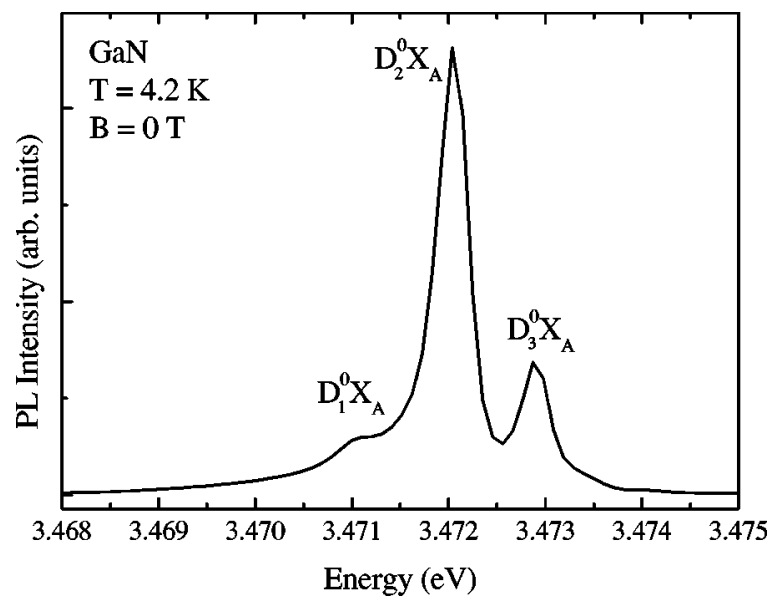

FIG. 1. The emission connected with bound exciton recombination measured at $4.2 \mathrm{~K}$.

could be expected for nearly unstrained GaN. ${ }^{15,11,16}$ Having this energy one can determine localization energies of the excitons bound to these donors as $7.9,6.9$, and $6.0 \mathrm{meV}$, respectively.

The emission spectrum in the region of the two-electron satellites (TES), close to $3.45 \mathrm{eV}$, for magnetic field $B=0$, is shown in Fig. 2. Two lines, $L 1$ at $3.4467 \mathrm{eV}$ and $L 2$ at $3.4477 \mathrm{eV}$, dominate the TES emission. In addition to these two lines there are two weak lines on the low-energy side: $L 0_{A}$ at $3.442 \mathrm{eV}$ and $L 0_{B}$ at $3.4433 \mathrm{eV}$. On the high-energy side there are several other, smaller lines indicating the presence of more than one donor involved in the two-electron excitation. Line $L 3$ consist of two closely spaced lines $L 3_{A}$, at $3.4494 \mathrm{eV}$ and $L 3_{B}$ at $3.4501 \mathrm{eV}$, the next line $L 4$ lies at $3.4514 \mathrm{eV}$. A weak line $L 5$ lies at $3.4530 \mathrm{eV}$, line $L 6$ is at $3.4542 \mathrm{eV}$, and line $L 7$ at $3.4562 \mathrm{eV}$. To identify transitions connected with two-electron excitation, three type of experiments have been performed. First, time-resolved measurements of the two-electron region and the bound-exciton region have been made. This experiment can show if a particular donor-bound exciton emission is correlated with a corresponding two-electron transition. The second experi-

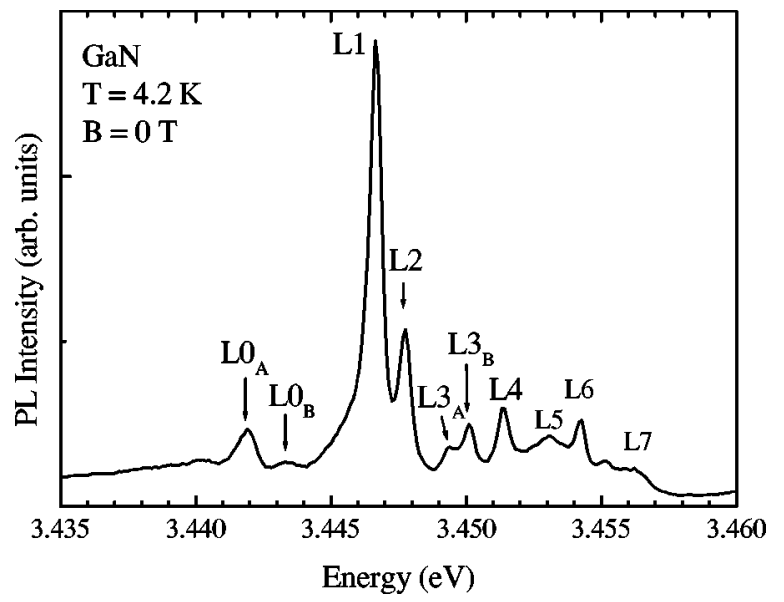

FIG. 2. The emission connected with two-electron satellite (TES) transitions measured at $4.2 \mathrm{~K}$.

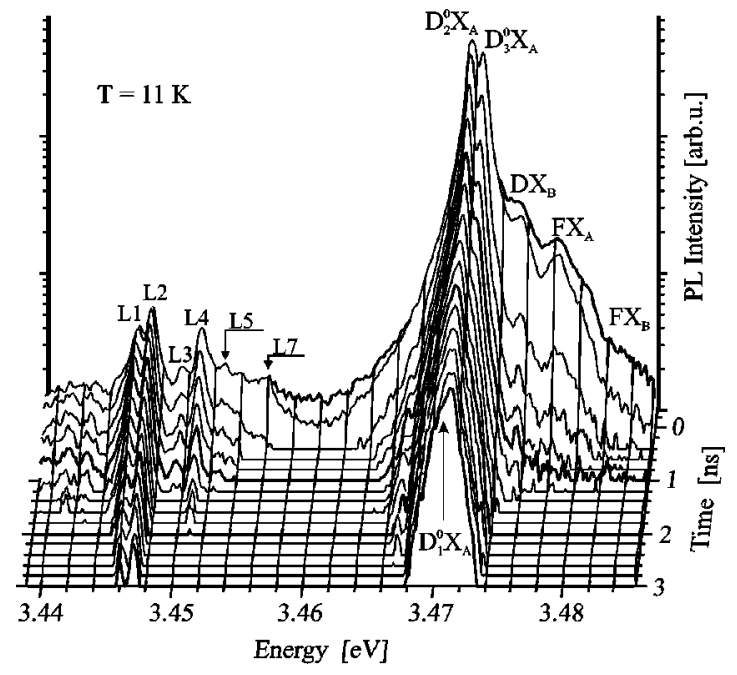

FIG. 3. Time-resolved spectra of freestanding $\mathrm{GaN}$ at $11 \mathrm{~K}$.

ment, the most important one, is magnetospectroscopy of the two electron transitions. The third experiment involves the temperature dependence of the two-electron emission.

\section{A. Time-resolved luminescence}

The dominant emission in the exciton region is due to excitons bound to neutral donors $\left(D^{0} X\right)$. The exciton emission lines are sharp, but in order to obtain good temporal resolution it is necessary to use a monochromator with a lower spectral resolution. Although the spectral resolution in the time-resolved measurements was lower than that in the magneto-optic measurements, it was still possible to distinguish sharp exciton lines. In this work, the most interesting spectral features are donor-bound exciton lines and their twoelectron satellites. The time-resolved spectra involving these features are shown in Fig. 3.

Photoluminescence transients of the donor-bound excitons and the two-electron transitions are shown in Fig. 4. The $D_{2}^{0} X_{A}$ line has a two-exponential decay. At the beginning its dynamics are faster and later on they slow down. It is possible that the long-lived tail of the $D_{2}^{0} X_{A}$ emission is connected with another weak but long-lived line. We think that this line may be connected with the $D_{1}^{0} X_{A}$ emission line, which is weak, and very close to the $D_{2}^{0} X_{A}$ line, so that it may not be possible to resolve it spectrally in the timeresolved measurements. However, the faster decay time $\left(t_{D 2}=0.5 \pm 0.1 \mathrm{~ns}\right)$ very well characterizes the $D_{2}^{0} X_{A}$ line.

The $D_{3}^{0} X_{A}$ line is weaker than the $D_{2}^{0} X_{A}$ line and has a faster recombination rate. Therefore, it was necessary to make corrections for the background coming from the $D_{2}^{0} X_{A}$ line. After the corrections, the $D_{3}^{0} X_{A}$ decay time, $t_{D 3}=0.31$ $\pm 0.03 \mathrm{~ns}$, has been obtained. The dynamics of a donorbound exciton line with a two-exponential decay has been reported previously, ${ }^{17,18}$ but up to now these emissions had not been resolved into separated components. The timeresolved spectra reveal that the shortest decay time corresponds to the donor having the exciton with the smallest localization energy. On the other hand the longest decay time 


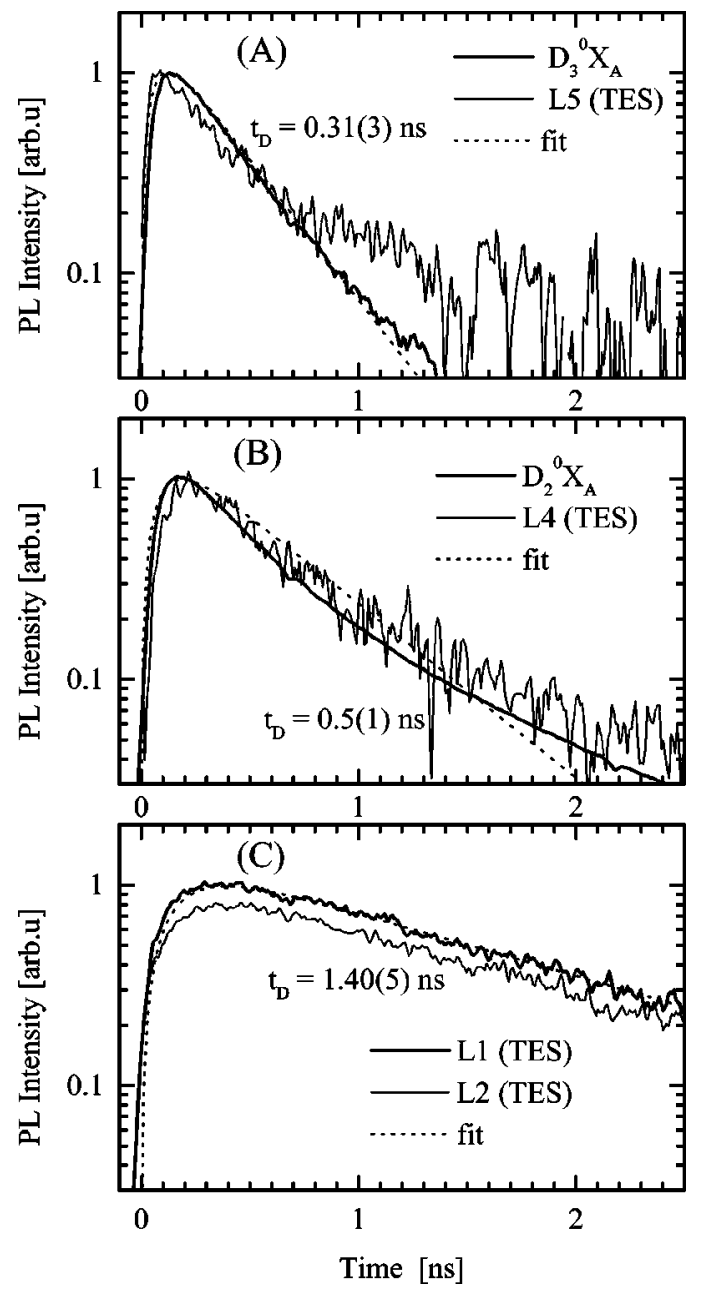

FIG. 4. PL transients of the most important lines (a) $D_{3}^{0} X_{A}$ and $L 5$ lines, (b) $D_{2}^{0} X_{A}$ and $L 4$ lines, (c) $L 1$ and $L 2$ lines. Thick lines denote direct recombination of the bound excitons; thin lines, PL in the TES region; and dotted lines, fits. All lines (except L2) are normalized to 1 .

corresponds to the donor whose exciton has the largest localization energy. This observation is in agreement with the theory proposed by Rashba et al. ${ }^{19}$

In the two-electron region, close to $3.45 \mathrm{eV}$, two relatively strong lines L1 and L2, at energies 3.4467 and $3.4477 \mathrm{eV}$, are observed. Weaker lines $L 3, L 4, L 5$, and $L 6$, follow them. A comparison of the dynamics of these lines shows that they can be arranged into three groups. The decay times of these groups are shown in Fig. 4.

The line L5 is relatively weak and its decay is relatively fast $\left(t_{D}\right.$ about $\left.0.3 \mathrm{~ns}\right)$. These dynamics are similar to those of the $D_{3}^{0} X_{A}$ line, which suggest that the $L 5$ line is connected with the TES of the $D_{3}^{0} X$ line. The $L 4$ and $L 3$ lines have dynamics similar to those of the $D_{2}^{0} X_{A}$ line, which corresponds to the bound exciton of localization energy $6.9 \mathrm{meV}$. This suggests that these lines are connected with the TES of $D_{2}^{0} X_{A}$. The PL transients of the $L 1$ and $L 2$ lines are monoexponential with decay time $t_{D}=1.40 \pm 0.05 \mathrm{~ns}$. Such a slow recombination rate can be compared with the recombination rate of the long-lived tail of the $D_{2}^{0} X_{A}$ line that is

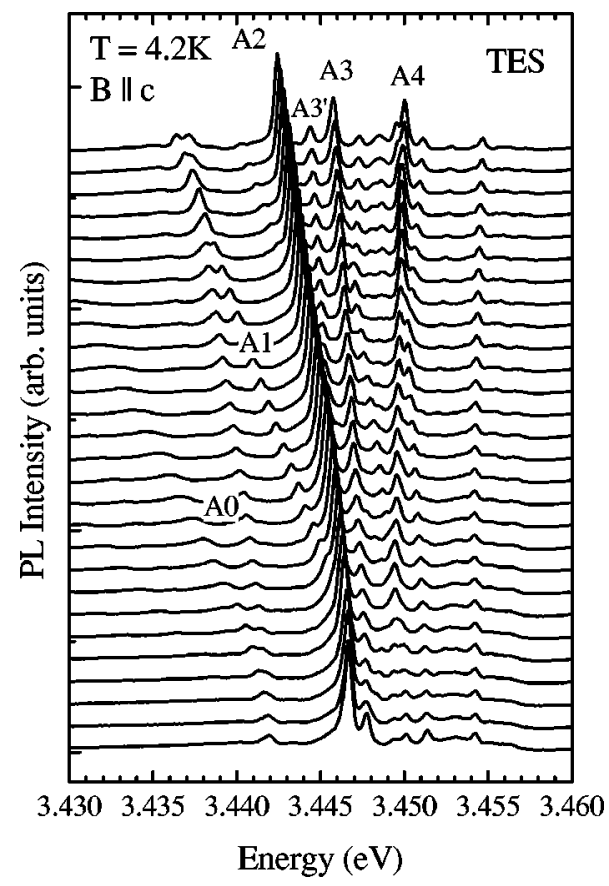

FIG. 5. The two-electron emission at $4.2 \mathrm{~K}$ in a magnetic field $B \| c$, for $B$ varying from 0 to $28 \mathrm{~T}$.

probably connected with the decay of the $D_{1}^{0} X_{A}$ line. Therefore, the most intense TES lines at 3.4467 and $3.4477 \mathrm{eV}$ have decay times corresponding to the decay time of the donor with the largest localization energy $(7.9 \mathrm{meV})$.

The decay times of the $L 6$ and $L 7$ lines are very short, comparable with those of $D^{0} X_{B}$ transients and of free excitons $\left(t_{D}=0.1-0.2 \mathrm{~ns}\right)$. Therefore, the $L 6$ line might be a TES replica of the $D^{0} X_{B}$ transition. The $L 7$ line possibly results from scattering of the free excitons on donors with simultaneous excitation of the donor to an excited state.

\section{B. Magnetospectroscopy of the two-electron satellites}

Two-electron satellites will split in a magnetic field into several components directly related to the splitting of excited states of the donor involved in these excitations. The twoelectron satellite luminescence in magnetic field $B$ parallel, and perpendicular to the c-axis, is shown in Figs. 5 and 6, respectively. The magnetic fields range from $B=0$ to $B$ $=28 \mathrm{~T}$.

From the time-resolved spectra, it is known that the two main TES lines, at 3.4467 and $3.4477 \mathrm{eV}$, are connected with the same donor. It is seen that these two lines split into at least three components for $B$ parallel to the $c$ axis and into four components for $B$ perpendicular. This splitting is similar to that found in homoepitaxial $\mathrm{GaN},{ }^{14}$ where excitations to $2 s, 2 p_{0}$, and to $2 p_{+}$states are observed for the $B \| c$ configuration. The excitation to the $2 p_{-}$state is very weak in this configuration. On the other hand, in the $B \perp c$ configuration, the excitation to $2 s, 2 p_{0}, 2 p_{+}$, and $2 p_{-}$states are observed, with the excitation to $2 p_{-}$the strongest one. For freestanding $\mathrm{GaN}$, the most intense line emerges from the $3.4477 \mathrm{eV}$ line and is present on the high-energy side of this line, as can be seen in Fig. 6. We assign this component as 


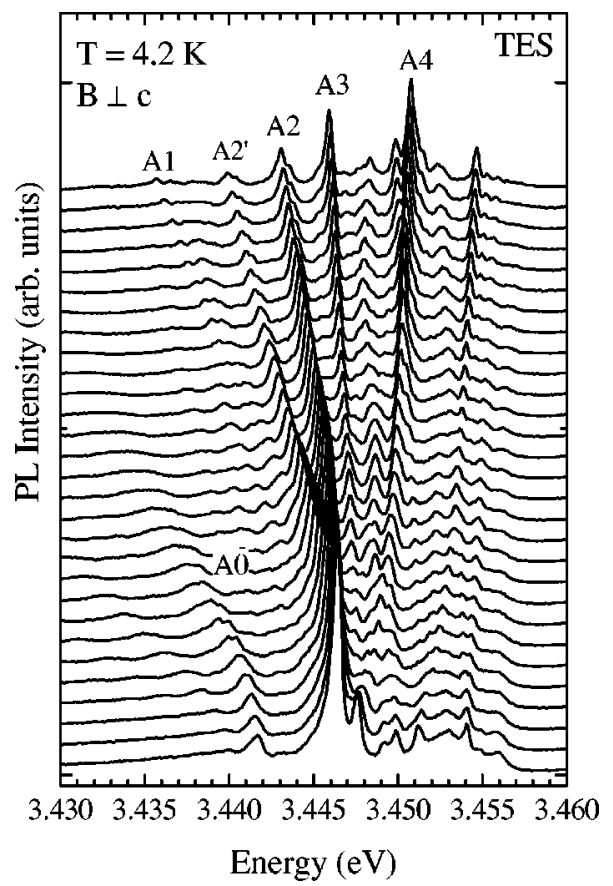

FIG. 6. The two-electron emission at $4.2 \mathrm{~K}$ for $B \perp c$, with $B$ varying from 0 to $28 \mathrm{~T}$.

due to excitation to the $2 p^{-}$state. The remaining excitations to the $2 p_{+}, 2 p_{0}$, and $2 s$ states can be identified for the perpendicular configuration of the magnetic field as well.

The energy difference between of the principal $D^{0} X$ transition, corresponding to the situation in which the donor is left in the ground state and its two-electron satellite line gives directly an intra-donor transition energy. Within the effective mass approximation the resulted excitation energies obtained from experimental data can be compared with calculation based on the theory describing a hydrogen atom in a magnetic field. ${ }^{20}$ In Fig. 7 the fit made for the excitations

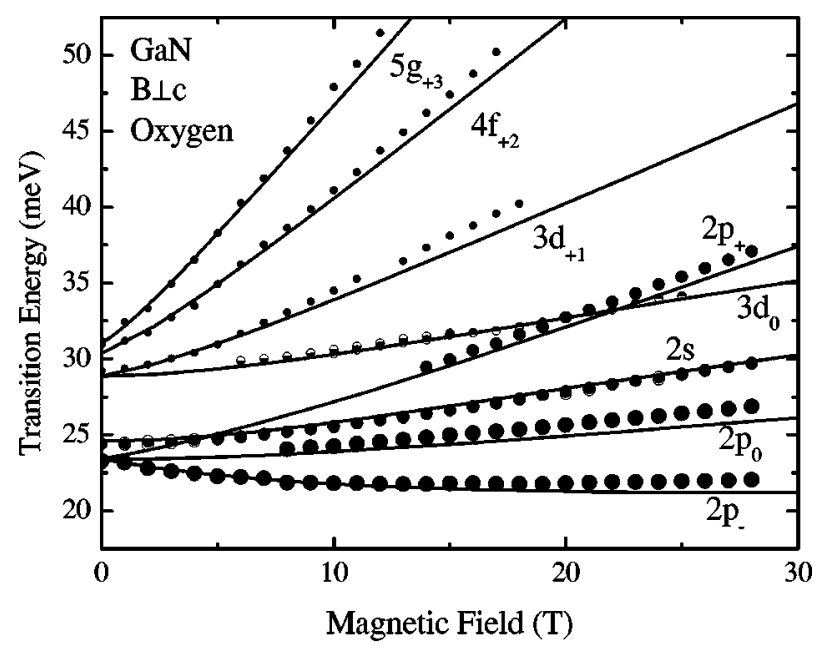

FIG. 7. Energies of excited states of the oxygen donor with respect to the $1 s$ ground state obtained from measurements (points) and compared with theoretical calculations (lines) based on a hydrogenlike model for the $B \perp c$ configuration. related to the donor responsible for the bound exciton with the largest $(7.9 \mathrm{meV})$ localization energy $\left(D_{1}^{0} X_{A}\right)$ for the $B \perp c$ configuration is shown.

The experimental points are taken with respect to the low spin component of the $D_{1}^{0} X_{A}$ transition. A set of lines marked in Fig. 6 as $A 0, A 1, A 2, A 3$, and $A 4$ has been fitted. The fit has been made for an effective Rydberg equal to 30.8 $\pm 0.5 \mathrm{meV}$, a cyclotron energy $h \omega_{\mathrm{c}}$ equal to $0.5412 \mathrm{meV} / \mathrm{T}$ (which corresponds to $m^{*}=0.222 m_{0}$ ), and a chemical shift at the $1 s$ state equal to $-1.5 \mathrm{meV}$. The fit is very good also for the higher excited states connected with $n=3,4$, and 5 . An excellent fit has been found for $3 d_{+1}, 4 f_{+2}$, and $5 g_{+3}$ states. Since the calculations given in Ref. 20 includes states only up to $n=4$, the fit to the $5 g+3$ state has been performed according to a calculation done by Rosner et al. ${ }^{21}$

The best fit to the $2 s$ donor state is obtained under the assumption that the $L 1$ line (TES of $D_{1}^{0} X_{A}$ at $B=0$ ) is the emission connected with the excitation to that state. The lines $A 2^{\prime}$ and $A 2$ observed in a magnetic field are connected with different spin directions of the $2 s$ electron in the final state. The spin splitting at the initial $D_{1}^{0} X_{A}$ state is independently experimentally detected and it agrees with the splitting of the excitation to the $2 s$ state. Therefore, the excitations for both spin components have been included in the experimental points in Fig. 7.

Since the best fit obtained for the $L 1$ line has connected it with an excitation to the $2 s$ state, therefore the $L 2$ line must be connected with an excitation to the $2 p$ donor state. The fit to the splitting of the $2 p$ state in a magnetic field, according to Ref. 20, is also shown in Fig. 7. The fit requires lowering of the $2 p$ state with respect to the $2 s$ state by $1 \mathrm{meV}$. This assignment suggests a positive chemical shift of the $2 s$ state. As it will be discussed later, the crystal field existing in GaN cannot cause this effect. In addition to lowering of the $2 p$ state, there is a systematic discrepancy in the description of all excitations to the $2 p$ states. This discrepancy, as seen in Fig. 7, increases linearly with magnetic field ( $0.035 \mathrm{meV} / \mathrm{T})$. The measured splitting between $2 p_{+}$and $2 p_{-}$states should equal the cyclotron energy, and indeed, it is in good agreement with theory.

A good fit is also obtained for the Faraday configuration $(B \| c)$ as shown in Fig. 10. The excitation to the $n=3$ state is well described by theory. The excitation to the $2 s$ state, as for the Voigt configuration $(B \perp c)$, is located above the excitation to the $2 p$ state. The splitting between excitations to the $2 p_{+}$and $2 p_{-}$states is equal to cyclotron energy. A line $2 p_{0}^{\prime}$, emerging from the $2 s$ state and parallel to $2 p_{0}$ state, is the most intriguing one. This line, marked in Fig. 5 as $A 3^{\prime}$, will be discussed later on.

Generally, it is fair to say that the description of the magnetic field dependence of the most intense TES lines, marked in Fig. 5 as A lines, is in agreement with theoretical predictions. As it will be discussed later on, we assign the $D_{1}$ donor as due to oxygen.

In addition to excitations connected with $D_{1}^{0} X_{A}$ (oxygenbound exciton), there are other weak TES seen in Fig. 6. These lines may be assigned as TES transitions connected with $D_{2}^{0} X_{A}$. The best fit for some of these lines, shown in 


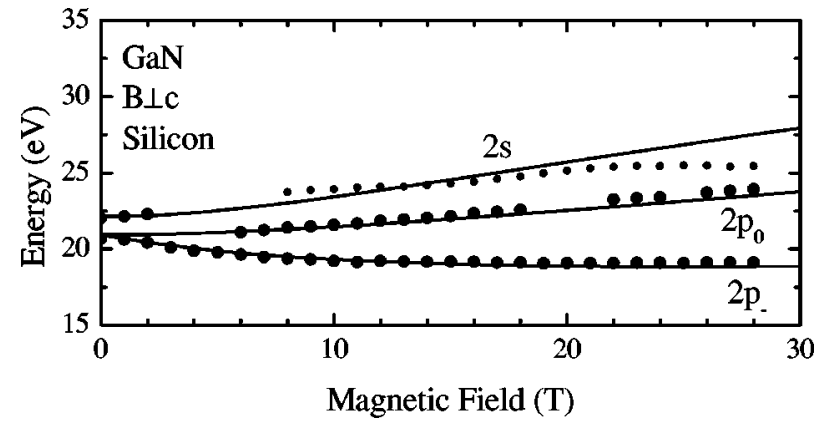

FIG. 8. Energies of $2 p_{0}, 2 s$, and $2 p_{-}$states with respect to the lower spin component of the $D_{2}^{0} X_{A}$ obtained from measurements in $B \perp c$ configuration (points) and compared with theoretical calculations (lines) based on a hydrogenlike model.

Fig. 8, was obtained assuming no chemical shift for this donor and using an effective Rydberg of $29.5 \mathrm{meV}$. Also in this case, the best fit is obtained for the $2 s$ state being above the $2 p$ state. We assign the donor responsible for these lines to silicon. The excitations connected with the donor having an exciton with a localization energy of $6.0 \mathrm{meV}\left(D_{3}^{0} X_{A}\right)$ are too weak to allow an analysis of the magnetic-field dependence.

\section{Temperature dependence of two-electron satellites}

The dependence of the TES emission spectra in the temperature range 4.2-15 $\mathrm{K}$ is shown in Fig. 9. The intensities of the two-electron transitions strongly depend on temperature. The intensity of the $3.4467 \mathrm{eV}$ line goes down, and the intensity of the $3.4477 \mathrm{eV}$ line goes up and reaches a maximum intensity at $11 \mathrm{~K}$. The analysis of the magnetic field splitting of the 3.4467 and $3.4477 \mathrm{eV}$ lines indicates that they are connected with excitations to $2 s$ and $2 p$ states, respectively. The same pattern of behavior can be observed for the lines $L 3_{B}$ and $L 4$ connected with the $D_{2}^{0} X_{A}$. The intensity of the $3.4501 \mathrm{eV}$ line $\left(L 3_{B}\right)$ decreases with increase of temperature. On the other hand, the intensity of the $3.4514 \mathrm{eV}$ line (L4) increases with increase of temperature up to $11 \mathrm{~K}$.

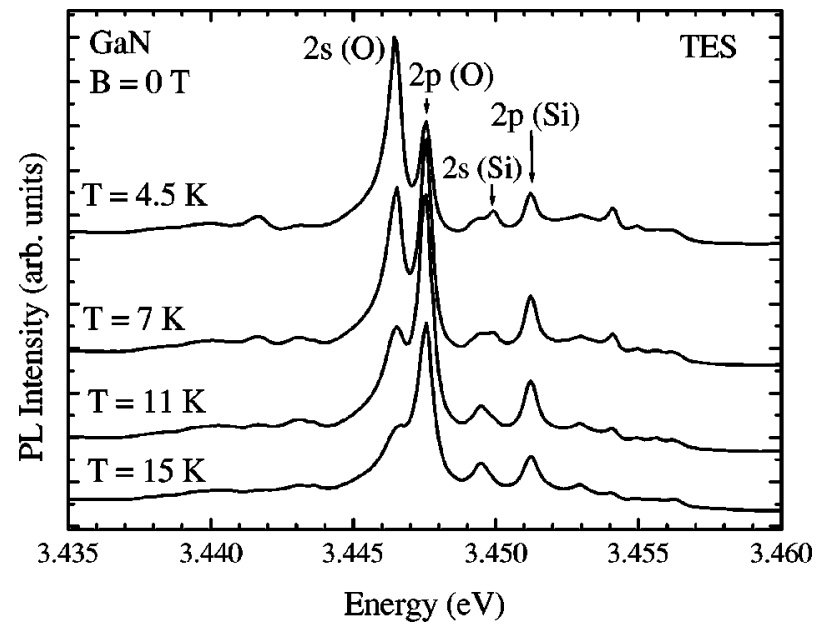

FIG. 9. Temperature dependence of the two-electron emission.

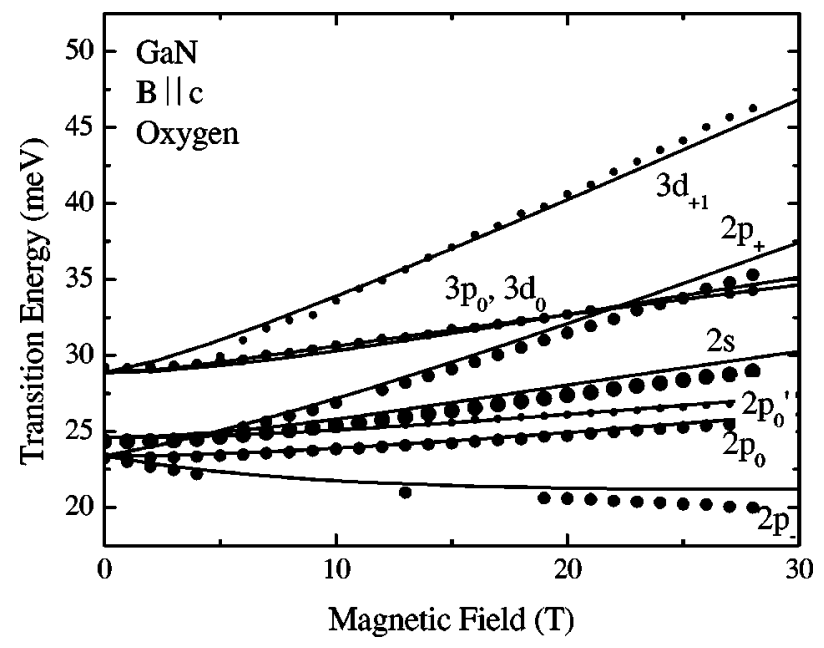

FIG. 10. Energies of excited states of the oxygen donor with respect to the $1 s$ ground state obtained from measurements (points) and compared with theoretical calculations (lines) based on a hydrogenlike model for the $B \| c$ configuration.

The increase of the $3.4477 \mathrm{eV}$ line is even stronger if one takes into account that in this temperature range, the intensities of all exciton lines decrease. A plot of normalized intensity of the $3.4477 \mathrm{eV}$ line (normalized with respect to the other TES lines) versus $1 / \mathrm{kT}$ gives an activation energy of 1 $\mathrm{meV}$. This result indicates that the initial state of the $D_{1}^{0} X_{A}$ is split into two components, separated by $1 \mathrm{meV}$. The excitation to the $2 s$ state goes from the ground state of $D_{1}^{0} X_{A}$, and the excitation to $2 p$ goes from the higher component of the $D_{1}^{0} X_{A}$ initial state.

As was mentioned earlier, the same situation exists with excitations of the other bound exciton, having a localization energy of $6.9 \mathrm{meV}\left(D_{2}^{0} X_{A}\right)$. The line $L 4$, interpreted as due to an excitation to $2 p$ states, is gaining intensity in the temperature range $4.5-11 \mathrm{~K}$. On the other hand, the excitations to the $2 s$ state are losing intensity in the same temperature range. Therefore, it is consistent with the magneto-optical results shown in Fig. 8 to assign the 3.4501 and $3.4514 \mathrm{eV}$ lines to excitations to $2 s$ and $2 p$ states of the $D_{2}^{0} X_{A}$, respectively.

\section{DISCUSSION}

The experimental results of time-resolved spectroscopy allow correlation of TES transitions with donors having different exciton localization energies. On the other hand, magneto-optical measurements allow us to identify emission lines in the 3.440-3.455 eV range as two-electron satellites. The magnitude of the splitting in a magnetic field, and the fit to the theory presented in Fig. 7 and Fig. 10, indicate that the excitations to $2 s, 2 p$, and higher excited states of the shallow donor are indeed observed. The calculated values of effective Rydbergs are in agreement with values reported for shallow donors in $\mathrm{GaN}^{22,23}$ However, the energy difference between the $2 p_{+}$and $2 p_{-}$states has been found to be the same for the $B \| c$ and $B \perp c$ configurations, which indicates that the electron effective mass anisotropy is negligible. This 
is contrary to our previous report in which a small anisotropy was detected in homoepitaxial GaN layers. ${ }^{14}$

The most intriguing result is that for both donors, the $2 s$ state is above the $2 p$ state. The same pattern of levels was reported previously for homoepitaxial layers. ${ }^{14}$ The crystal field present in GaN cannot cause such an upward shift of the $2 s$-state energy. The crystal field should predominantly split $2 p$ states, leaving the $2 s$ state lying between $2 p_{0}$ and the pair of $2 p_{+}$and $2 p_{-}$. Therefore, the energy shift of the $2 s$ state seems to be due to a positive chemical shift of this state. Such a positive chemical shift has also been postulated for a donor observed in the TES found in homoepitaxial GaN. ${ }^{14}$ However, a positive chemical shift, while possible is principle, should be very rare. To observe a positive chemical shift on several donors with different ionization energies in GaN seems to be unlikely. Also, the temperature dependence of the TES cannot be explained by a positive chemical shift.

There is also a discrepancy between the $1 s-2 p$ excitation energy observed in optical absorption, and the same energy found from TES measurements. Freestanding $\mathrm{GaN}$ has been investigated in the far infrared, and two residual donors $\mathrm{O}$ and Si have been identified. ${ }^{24,25}$ It has been found that $1 s-2 p$ transitions are taking place at $26 \mathrm{meV}$ for $\mathrm{O}$, and at $23 \mathrm{meV}$ for $\mathrm{Si}^{25}$ From our measurements, energy differences between the $1 \mathrm{~s}$ and $2 \mathrm{~s}$ states have been found to be equal to 24.3 and $21.9 \mathrm{meV}$ for the donors connected with the $D_{1}^{0} X_{A}$ and $D_{2}^{0} X_{A}$ lines, respectively. The freestanding sample investigated here has been grown by the same technology as that reported in Ref. 14, and contamination by the same residual impurities should be present. Therefore, in spite of slightly different optical and TES energies, it is possible to assign the donor connected with the exciton of localization energy 7.9 $\mathrm{meV}\left(D_{1}^{0} X_{A}\right)$ as due to oxygen, and the second donor (responsible for the $D_{2}^{0} X_{A}$ line) as due to silicon.

In the case of the O-related donor, the difference between the optically measured $1 s-2 p$ transition at $26 \mathrm{meV}$ and the $1 s-2 s$ energy deduced from the TES $(24.3 \mathrm{meV})$ may be connected with a lattice relaxation due to coupling of electronic states to lattice vibrations. The $1 s$ and $2 s$ states, due to different extensions of their wavefunctions, may be coupled to lattice vibrations in a different way. The TES excitations are connected with transfer between minima, in a configuration coordinate diagram. On the other hand, the optical transition is a direct one and will take place at higher energy. In the case of the TES of the $D_{2}^{0} X_{A}$, the $1 s-2 p$ and $1 s-2 s$ transitions have energies 20.6 and $21.9 \mathrm{meV}$, respectively. The difference between them, $1.3 \mathrm{meV}$, can be accounted for by the splitting of the initial $D_{2}^{0} X_{A}$ state. The excitation to the $2 s$ state at $21.9 \mathrm{meV}$ can be compared with the optical transition $1 s-2 p$ observed in far infrared for Si impurity at 23 $\mathrm{meV}$. The difference between 21.9 and $23 \mathrm{meV}$ can be due to lattice relaxation, as in the case of $\mathrm{O}$ impurity.

The energies of the $1 s-2 p$ and $1 s-2 s$ excitations found in homoepitaxial GaN layers at 21.2 and $22.4 \mathrm{meV}^{14}$ respectively, are close to the excitation energies observed for the $\mathrm{Si}$ impurity in freestanding GaN. Therefore the main dominant residual donor in homoepitaxial $\mathrm{GaN}$ can be assigned to Si.

The consistent interpretation of the magneto-optical data and the temperature dependence of the TES shows that the initial state of the $D_{1}^{0} X_{A}$ is split by $1.0 \mathrm{meV}$. In the case of $D_{2}^{0} X_{A}$, the splitting of the initial state is of the same order $(1.3 \mathrm{meV})$. The initial state of the exciton bound to a neutral donor contains two electrons and one hole. Electronic exited states of this system connected with excitations within the donor or the exciton would take place at an energy of about $20 \mathrm{meV}$. Thus, within the model of pure electronic states it is not possible to explain the experimental TES data.

The possible explanation lies in the rotational states of the bound exciton. Such states have been postulated previously for CdS (Ref. 26) and CdTe. ${ }^{27}$ Experimental evidences of the presence of the donor bound exciton rotational states in $\mathrm{GaN}$ have been already reported. ${ }^{28,29}$ However, there is no theory for rotational states in $\mathrm{GaN}$ and thus it is not possible to estimate the rotational excitation energy. Also, it is difficult to explain why TES excitation to the $2 p$ state should take place predominantly from the first exited rotational state of the $D_{1}^{0} X_{A}$ system.

It is interesting to note that TES excitations from the ground state of the $D_{1}^{0} X_{A}$ are taking place to $2 \mathrm{~s}, 3 d_{+1}$, $4 f_{+2}$, and $5 g_{+3}$ states. The characteristic feature of $3 d_{+1}$, $4 f_{+2}$, and $5 g_{+3}$ states is that their wave functions are extended along the magnetic field direction. In addition to these excitations, it seems that the one from the ground state of the $D_{1}^{0} X$ to the $2 p_{0}$ (the wave function of $2 p_{0}$ is extended along the field direction) takes place as well. The line $A 3^{\prime}$ shown in Fig. 5 it seems to be responsible for this transition. The calculated excitation to $2 p_{0}$ from the ground state is indicated in Fig. 10 as the $2 p_{0}$, one. Therefore, there are two excitations to the $2 p_{0}$ state the first one from the ground one and the second one from the first excited state of $D_{1} X_{A}$ indicated in Fig. 10 as $2 p_{0}$, and $2 p_{0}$, respectively.

\section{CONCLUSIONS}

Freestanding $\mathrm{GaN}$ has been investigated by time-resolved photoluminescence, magneto-spectroscopy, and temperature dependence of two-electron satellites (TES). It has been found that an exciton bound to the deeper donor is characterized by a longer decay time. The longest lifetime $t$ $=1.40 \pm 0.05 \mathrm{~ns}$ has been determined for the TES of $D_{1}^{0} X_{A}$ (exciton $A$ bound to oxygen). Differences in decay times of the various bound excitons allow correlation of the TES with particular donor-bound exciton lines.

The most intense TES transitions have been observed for the deepest donor, also the one with the highest exciton localization energy, assigned to oxygen. The magneto-optic experiments reveal a rich spectrum of spin-split lines. Analysis of the TES in magnetic fields shows that excitations of the oxygen donor to states of $n=2,3,4$, and 5 have been observed. Splitting of excited states of the neutral oxygen donor is well described by the theory of a hydrogen atom in a magnetic field. However, a systematic lowering of the excitation energy of the $2 p$ state with respect to that of the $2 \mathrm{~s}$ state, for both the $\mathrm{O}$ and $\mathrm{Si}$ donors (by 1.0 and $1.3 \mathrm{meV}$, 
respectively) has been found. The temperature investigation of the TES transitions reveals that this effect is connected with a splitting of the $D_{1}^{0} X_{A}$ and $D_{2}^{0} X_{A}$ initial states.

The nature of the splitting of the initial states of the donor-bound exciton is discussed. It is argued that this effect may be caused by the presence of an excited state due to a rotational degree of freedom of the donor-bound exciton system.

*Electronic address: wysmolek@fuw.edu.pl

${ }^{1}$ D. C. Look and J. R. Sizelove, Appl. Phys. Lett. 79, 1133 (2001).

${ }^{2}$ D. C. Reynolds, D. C. Look, B. Jogai, A. W. Saxler, S. S. Park, and J. Y. Han, Appl. Phys. Lett. 77, 2879 (2000).

${ }^{3}$ E. Oh, S. K. Lee, S. S. Park, K. Y. Lee, I. J. Song, and J. Y. Han, Appl. Phys. Lett. 78, 273 (2001).

${ }^{4}$ P. Dean, J. D. Cuthbert, D. G. Thomas, and R. Lynch, Phys. Rev. Lett. 18, 122 (1967)

${ }^{5}$ D. C. Reynolds, C. W. Litton, and T. C. Collins, Phys. Rev. 174, 845 (1968).

${ }^{6}$ D. C. Reynolds, C. W. Litton, and T. C. Collins, Phys. Rev. 177, 1161 (1969).

${ }^{7}$ D. C. Reynolds and T. C. Colins, Phys. Rev. 185, 1099 (1969).

${ }^{8}$ D. C. Reynolds, C. W. Litton, R. J. Almassy, S. B. Nam, P. J. Dean, and R. C. Clarke, Phys. Rev. B 13, 2507 (1976).

${ }^{9}$ B. J. Skromme, H. Zao, B. Goldenberg, H. S. Kong, M. T. Leonard, G. E. Bulman, C. R. Abernathy, and S. J. Pearton, in III-IV Nitrides, edited by F. A. Ponce et al., Mater. Res. Soc. Symp. Proc. No. 449 (Materials Research Society, Pittsburgh, 1997), p. 713.

${ }^{10}$ A. Fiorek, J. M. Baranowski, A. Wysmolek, K. Pakula, and M. Wojdak, Acta Phys. Pol. A 92, 742 (1997).

${ }^{11}$ K. Kornitzer, T. Ebner, K. Thonke, R. Sauer, C. Kirchner, V. Schwegler, M. Kamp, M. Leszczynski, I. Grzegory, and S. Porowski, Phys. Rev. B 60, 1471 (1999).

${ }^{12}$ J. M. Baranowski, Z. Liliental-Weber, K. Korona, K. Pakula, R. Stepniewski, A. Wysmolek, I. Grzegory, G. Nowak, S. Porowski, B. Monemar, in III-V Nitrides, edited by F. A. Ponce et al., Mater. Res. Soc. Symp. Proc. No. 449 (Materials Research Society, Pittsburgh, 1997), p. 393.

${ }^{13}$ A. Wysmolek V. F. Sapega, T. Ruf, M. Cardona, M. Potemski, P. Wyder, R. Stepniewski, K. Pakula, J. M. Baranowski, I. Grzegory, et al., Proceedings of the International Workshop on Nitride Semiconductors 2000, IPAP Conf. Series No. 1 (IPAP, Tokyo, 2000), p. 579.

\section{ACKNOWLEDGMENTS}

This work has been partially supported by State Committee for Research (Republic of Poland), Grant No. 2P03B 011 22, NATO Science Program, EC Project No. HPRI-CT-199940013. The work of DCL was supported under U.S. Air Force Contract No. F33615-00-C-5402, and AFOSR Grant No. F49620-00-1-0347.

${ }^{14}$ M. Wojdak, J. M. Baranowski, A. Wysmolek, K. Pakuła, R. Stępniewski, M. Potemski, I. Grzegory, and S. Porowski, in GaN and Related Compounds, edited by J. E. Northup et al., Mater. Res. Soc. Symp. Proc. No. 693 (Materials Research Society, Pittsburg, 2002), p. 18.10.

${ }^{15}$ R. Stępniewski, K. Korona, A. Wysmolek, J. Baranowski, K. Pakula, M. Potemski, G. Martinez, I. Grzegory, and S. Porowski, Phys. Rev. B 56, 15151 (1997).

${ }^{16}$ B. Gil, O. Briot, and R. Aulombard, Phys. Rev. B 52, R17 028 (1995).

${ }^{17}$ J. P. Bergman, B. Monemar, H. Amano, I. Akasaki, T. Detchprohm, K. Hiramatsu, and N. Sawaki, Proceedings of ICSCRM'95, Kyoto 1995, IOP Conference Procedings No. 142, (IOP, Bristol, 1996), p. 931.

${ }^{18}$ K. P. Korona, J. Kuhl, and J. M. Baranowski, Phys. Status Solidi B 215, 53 (1999).

${ }^{19}$ E. I. Rashba and G. E. Gurgenishvili, Sov. Phys. Solid State 4, 759 (1962).

${ }^{20}$ P. C. Macado and N. C. McGill, J. Phys. C 19, 873 (1986).

${ }^{21}$ W. Rosner, G. Wunner, H. Herold, and H. Ruder, J. Phys. B 17, 29 (1984).

${ }^{22}$ F. Mireles and S. E. Ulloa, Appl. Phys. Lett. 74, 248 (1999).

${ }^{23}$ H. Wang and A. B. Chen, Appl. Phys. Lett. 87, 248 (2000).

${ }^{24}$ W. J. Moore, J. A. F. Jr., G. C. B. Braga, R. J. Molnar, S. K. Lee, K. Y. Lee, and I. J. Song, Appl. Phys. Lett. 79, 2570 (2001).

${ }^{25}$ W. J. Moore, J. A. Freitas, S. K. Lee, S. S. Park, and J. Y. Han, Phys. Rev. B 65, 081201 (2002).

${ }^{26}$ C. H. Henry and K. Nassau, Phys. Rev. B 2, 997 (1970).

${ }^{27}$ C. B. a la Guillame and P. Lavallard, Phys. Stat. Solidi B 70, K143 (1975).

${ }^{28}$ B. J. Skromme, MRS Internet J. Nitride Semicond. Res. 4, 15 (1999).

${ }^{29}$ G. Neu, M. Teisseire, E. Frayssinet, W. Knap, M. L. Sadowski, A. M. Witowski, K. Pakula, M. Leszczynski, and P. Prystawko, Appl. Phys. Lett. 77, 1348 (2000). 